\title{
EDITORIAL
}

\section{Sanofi-Cell Research outstanding paper award of 2019}

Cell Research (2020) 30:941; https://doi.org/10.1038/s41422-020-00425-1

We are pleased to announce winners of the 2019 Sanofi-Cell Research Outstanding Research Article Award: Drs Chunru Lin and Liuqing Yang, ${ }^{1}$ for their paper entitled "LncRNAs-directed PTEN enzymatic switch governs epithelial-mesenchymal transition"; Drs Yan Shi, Shichun Lu, Hongkui Deng, ${ }^{2}$ for their paper entitled "A two-step lineage reprogramming strategy to generate functionally competent human hepatocytes from fibroblasts"; and Drs Keiichiro Suzuki and Juan Carlos Izpisua Belmonte, ${ }^{3}$ for their paper entitled "Precise in vivo genome editing via single homology arm donor mediated intron-targeting gene integration for genetic disease correction". Each award consists of a prize of $€ 5000$ sponsored by Sanofi.

Although PTEN has been considered one of the most important tumor suppressors, PTEN also plays important roles during homeostasis and pathological conditions. In the first award-winning research article, published in the April 2019 issue, Drs Chunru Lin, Liuqing Yang and colleagues ${ }^{1}$ showed that high glucose and growth factors including TGF- $\beta$, CTGF, SHH, and IL- 6 triggered the expression of a IncRNA, which bound an E3 ligase and facilitated its ligase activity towards PTEN, leading to the K27-linked polyubiquitination of PTEN. PTEN ${ }^{\mathrm{K} 27-p o l y U b}$ removed phospho-groups from serine/threonine residues in substrates including TWIST1, SNAI1, and YAP1, resulting in accumulation of these master regulators of EMT. This study revealed an unappreciated dimension of PTEN function as a protein serine/threonine phosphatase in physiological homeostasis and disease development. Direct lineage reprogramming to generate various human cell types in a straightforward manner in vitro has been reported, which is however, hindered by incomplete conversion with residual initial cell identity and limited function of target cells. In the second award-winning research article, published in the September 2019 issue, Drs Yan Shi, Shichun Lu, Hongkui Deng and colleagues $^{2}$ developed a new two-step strategy mimicking a natural cell-fate conversion route during regeneration to generate a large amount of functional competent hepatocytes in vitro. This strategy may become a general path to generating other mature cell types in vitro. Direct gene modification in living organisms by in vivo targeted genome-editing is a powerful tool for basic biology and treating inherited diseases. In the third award-winning research article, published in the October 2019 issue, Drs Keiichiro Suzuki, Juan Carlos
Izpisua Belmonte and colleagues ${ }^{3}$ described a versatile in vivo gene knock-in method, termed "Single homology Arm donor mediated intron-Targeting Integration (SATI)". This paper showed that SATI, which enables the targeting of a broad range of mutations and cell types, is a powerful genetic tool for in vivo genome editing.

Please join us to congratulate Drs Lin and Yang, Drs Shi, Lu and Deng, and Drs Suzuki and Izpisua Belmonte on their winning of the 2019 Sanofi-Cell Research Outstanding Paper Award.

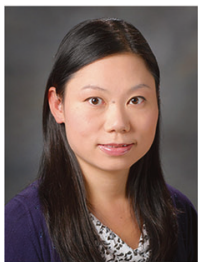

Dr Chunru LI
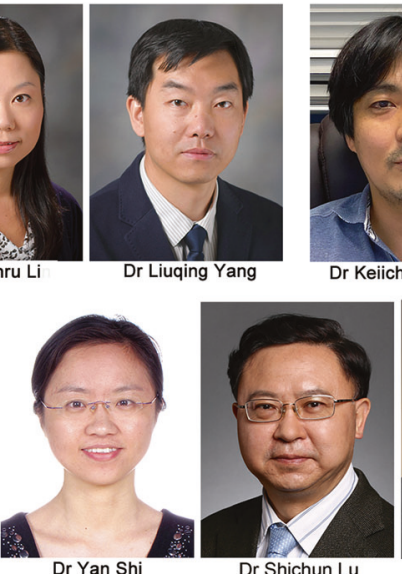

Dr Shichun Lu

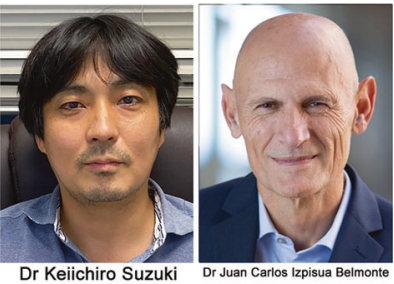

Dr Keiichiro Suzuki

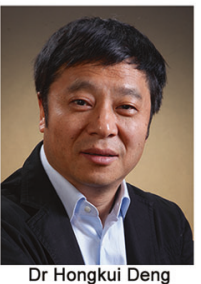

Cell Research Editorial Team ${ }^{1}$

${ }^{1}$ CAS Center for Excellence in Molecular Cell Science/Shanghai Institute of Biochemistry and Cell Biology, Chinese Academy of Sciences, Shanghai 200031, China Correspondence: (cellres@sibcb.ac.cn)

\section{REFERENCES}

1. Hu, Q. et al. Cell Res 29, 286-304 (2019).

2. Xie, B. et al. Cell Res 29, 696-710 (2019).

3. Sukuzi, K. et al. Cell Res 29, 804-819 (2019). 